\title{
Knockdown of RAB25 promotes autophagy and inhibits cell growth in ovarian cancer cells
}

\author{
YINGTAO LIU $^{1}$, XIANG TAO ${ }^{1}$, LUOQI JIA ${ }^{1}$, KWAI WA CHENG ${ }^{2}$, \\ YILING LU ${ }^{2}$, YINHUA YU ${ }^{1}$ and YOUJI FENG ${ }^{1,3}$ \\ ${ }^{1}$ Obstetrics and Gynecology Hospital of Fudan University, Shanghai 200011, P.R. China; ${ }^{2}$ Department of Systems Biology, \\ University of Texas, M. D. Anderson Cancer Center, Houston, TX 77054, USA; \\ ${ }^{3}$ Department of Obstetrics and Gynecology, Shanghai First People's Hospital, \\ Jiao Tong University, Shanghai 200080, P.R. China
}

Received March 23, 2012; Accepted August 6, 2012

DOI: $10.3892 / \mathrm{mmr} .2012 .1052$

\begin{abstract}
RAB25 belongs to the Rab family of small GTPases and is implicated in the development of various types of human cancer. To evaluate the role of RAB25 in ovarian cancer, RAB25 was knocked down by siRNA in HEY and ES-2 human ovarian cancer cells. Autophagy, cell growth and cell apoptosis were evaluated. The results showed that knockdown of RAB25 increased acidic vesicle organelles and GFP-microtubule-associated protein 1 light chain 3 punctate fluorescence in ovarian cancer cells. Autophagy that promoted by knockdown of RAB25 was not observed in cells where the ERK1/2 signaling pathway had been inhibited by U0126. Knockdown of RAB25 reduced cell cycle progression and cell growth. Apoptosis of ovarian cancer cells could be induced by knockdown of RAB25. These results support the tumorigenic role of RAB25 in ovarian cancer cells.
\end{abstract}

\section{Introduction}

Ovarian cancer is the second most common gynecological malignancy and affects more than 200,000 women worldwide each year. The symptoms of ovarian cancer are non-specific and therefore two-thirds of cases are not diagnosed until the later stages (1). Aggressive surgical reduction and new chemotherapeutic agents have improved the prognosis of advanced ovarian cancer $(2,3)$; however, the 5-year survival rate remains low in more than half of diagnosed women (4-8). Therefore, it is critical to investigate the biological behavior of ovarian

Correspondence to: Professor Youji Feng, Department of Gynecology, Obstetrics and Gynecology Hospital of Fudan University, 419 Fang Xie Road, Shanghai 200011, P.R. China E-mail: rab25.fsh@hotmail.com

Key words: RAB25, ovarian cancer, autophagy, extracellular signal-regulated kinases, proliferation, apoptosis cancer cells and identify new prognostic factors and therapeutic targets.

RAB25 belongs to the Rab family of small GTPases, which regulates various aspects of membrane recycling and trafficking to the plasma membrane (9). Unlike the other members of the ubiquitously expressed RAB11 sub-family, RAB25 expression is restricted to epithelial tissue (10) and is correlated with several epithelial cancers. Overexpression of RAB25 has been reported in liver (10) and bladder cancer (11) and is also associated with the aggressive behavior of ovarian and breast cancers $(12,13)$. RAB25 has been implicated in the promotion of cell proliferation, evasion of apoptosis and acceleration of in vivo tumor growth in ovarian cancer $(12,14,15)$.

Autophagy refers to the process of autodigestion in which the cell's own components are degraded and recycled by the lysosomal machinery (16). Autophagy is induced by starvation, hypoxia and high temperature. Through the partial digestion of cell components, autophagy provides nutrients that are necessary to maintain cell viability and prolonged survival (17). However, autophagy may result in the destruction of vital organelles, leading to cell death (18). Previous studies have suggested a correlation between the decline in autophagic activity and tumorigenesis $(19,20)$, while chemotherapeutic agents stimulate autophagic cell death $(21,22)$.

The role of RAB25 in ovarian cancer cells was evaluated in vitro. The present study reveals that knockdown of RAB25 by siRNA promoted autophagy through activation of the ERK1/2 signaling pathway. It indicated that knockdown of RAB25 resulted in the inhibition of cell proliferation and the induction of apoptosis. These results support the tumorigenic role of RAB25 in ovarian cancer cells.

\section{Materials and methods}

Cell culture and treatment. HEY and ES-2 human ovarian cancer cell lines (donated by the University of Texas, M. D. Anderson Cancer Center, Houston, TX, USA) were grown in RPMI-1640 medium (Gibco, Grand Island, NY, USA) supplemented with $100 \mathrm{IU} / \mathrm{ml}$ penicillin, $100 \mu \mathrm{g} / \mathrm{ml}$ streptomycin and $10 \%$ FBS (Hyclone, Logan, UT, USA) in a humidified 
atmosphere of $5 \% \mathrm{CO}_{2}$ at $37^{\circ} \mathrm{C}$. These cells were sub-cultured by adding $0.05 \%$ trypsin- $0.01 \%$ EDTA (Gibco) when the cells reached $80 \%$ confluence. For experiments involving the pharmacological inhibitor, the cells were serum-starved for $12 \mathrm{~h}$ and then treated with U0126 (Sigma Aldrich, St. Louis, MO, USA) at a concentration of $10 \mu \mathrm{M}$ for $24 \mathrm{~h}$. Cells treated with DMSO (Sigma Aldrich) served as the control.

siRNA transfection. The RAB25 ON-TARGET plus SMART pool siRNA (siRab) and siGLO non-targeting siCONTROL siRNA (siNon) were purchased from Dharmacon (Lafayette, CO, USA). Cells were transfected with siRab or siNon using DharmaFCET 1 reagent (Dharmacon) according to the manufacturer's instructions. Briefly, the siRNA and transfection reagent were diluted in serum-free Opti-MEM and mixed. Following incubation at room temperature for $20 \mathrm{~min}$, the mixture was added to the cells at a final siRNA concentration of $50 \mathrm{nM}$. Following incubation for $6 \mathrm{~h}$, FBS was added to achieve a final concentration of $10 \%$ and the cells were incubated for $24 \mathrm{~h}$ prior to subsequent treatment. Cells treated with DharmaFECT 1 reagent served as the control.

RNA extraction and quantitative real-time PCR. Total RNA was extracted from HEY and ES-2 cells with TRIzol reagent (Invitrogen, Carlsbad, CA, USA) according to the manufacturer's instructions. Reverse transcription was performed using the Oligo (dT) 18 primer according to the RevertAid First-Strand cDNA Synthesis kit protocol (Fermentas, Vilnius, Lithuania). Quantitative PCR was performed with the SYBR Green Premix Ex Taq kit (Takara Bio, Inc., Dalian, China), which consisted of $2 \mu \mathrm{l}$ cDNA template, $10 \mu \mathrm{l} \mathrm{SYBR-Green}$ Real-time PCR Master mix and $0.2 \mu \mathrm{M}$ forward and reverse primers in a final volume of $20 \mu \mathrm{l}$. The primer sequences were as follows: RAB25 sense, 5'-GCCCTGGACTCTACCAAT GTTGA-3'; RAB25 antisense, 5'-GCTGTTCTGTCTCTGCTT GGACAC-3'; GAPDH sense, 5'-GCACCGTCA AGGCTGAGA AC-3'; and GAPDH antisense, 5'-TGGTGAAGACGCCAG TGGA-3'. The reactions were carried out with an ABI PRISM 7000 Sequence Detection System (Applied Biosystems, Foster City, CA, USA) for 40 cycles $\left(95^{\circ} \mathrm{C}\right.$ for $5 \mathrm{sec}, 60^{\circ} \mathrm{C}$ for $\left.31 \mathrm{sec}\right)$ after an initial $10 \mathrm{sec}$ of incubation at $95^{\circ} \mathrm{C}$. The fold change in the expression of each gene was calculated using the $-\Delta \Delta \mathrm{Ct}$ method and GAPDH was used as the internal control.

Western blot analysis. Cultured cells were washed with PBS and then lysed with RIPA solution (Shanghai Bocai Bio. Technology Co., Ltd., Shanghai, China). The lysate was cleared by centrifugation at $12,000 \mathrm{rpm}$ for $30 \mathrm{~min}$ and the protein was then quantified using the bicinchoninic acid (BCA) (Shanghai Bocai Bio. Technology Co., Ltd.) method according to the manufacturer's instructions. Proteins (30 $\mu \mathrm{g} /$ lane) were resolved by SDS-PAGE and transferred onto a PVDF membrane (Millipore, Billerica, MA, USA). Following blocking with 5\% BSA-TBST for $1 \mathrm{~h}$ at room temperature, the membrane was incubated with the primary antibodies overnight at $4^{\circ} \mathrm{C}$. The membrane was then incubated with HRP-conjugated secondary antibodies (Kangchen Bioengineering Corp., Shanghai, China; 1:5,000) or IRDye 700DX conjugated affinity goat anti-mouse IgM $(1: 2,500)$ for $1 \mathrm{~h}$ at $37^{\circ} \mathrm{C}$. Protein bands were visualized by ECL (Perkin
Elmer, Waltham, MA, USA) or by Odyssey infrared imaging system (Li-Cor, Lincoln, NE, USA), with GAPDH as a loading control. The mouse anti-GAPDH monoclonal antibody (1:5,000) was purchased from Kangchen Bioengineering Corporation; mouse anti-RAB25 monoclonal antibodies (1:500) were purchased from Abcam Inc. (Cambridge, MA, USA); rabbit anti-survivin polyclonal antibody $(1: 1,000)$ was purchased from R\&D Systems, Inc. (Minneapolis, MN, USA); rabbit anti-LC3B (1:1000), rabbit anti-Beclin 1 (1:1000), mouse anti-cyclin D1 (1:2000), mouse anti-cyclin B1 (1:2000), rabbit anti-cleaved-caspase-3 (1:1000) and rabbit antiphospho-ERK1/2 (1:2000) antibodies were purchased from Cell Signaling Technology, Inc. (Danvers, MA, USA).

Immunocytofluorescence. Cells were seeded on a cover glass and cultured in Opti-MEM for $24 \mathrm{~h}$. Adherent cells were fixed with $95 \%$ ethanol at $4{ }^{\circ} \mathrm{C}$ for $20 \mathrm{~min}$ and then incubated with $0.5 \%$ Triton-X 100 (Sangon, Shanghai, China) for $15 \mathrm{~min}$. Cells were blocked by adding goat serum blocking buffer (Mingrui, Shanghai, China) at room temperature for $30 \mathrm{~min}$ and then incubated with rabbit anti-RAB25 (Cell Signaling Technology, Inc.) primary antibody (1:500) at $4{ }^{\circ} \mathrm{C}$ overnight. The following day, cells were washed three times with PBS and incubated with Northern Lights anti-mouse IgG-NL557 (R\&D systems Inc.; $1: 200)$ at $37^{\circ} \mathrm{C}$ for $1 \mathrm{~h}$ in the dark. Following mounting with VECTASHIELD mounting medium with DAPI (Vector Laboratories, Burlingame, CA, USA), the cell images were acquired using DP 7.0 software and an Olympus BX51 fluorescence microscope.

Acridine orange staining assay. The formation of acidic vesicle organelles (AVOs) is a characteristic of autophagic cell death (23). To stain AVOs in cells, acridine orange (Sangon) was added into the medium at a final concentration of $1 \mu \mathrm{g} / \mathrm{ml}$. The cells were incubated at $37^{\circ} \mathrm{C}$ for $15 \mathrm{~min}$ and then harvested with trypsin and immediately analyzed on a flow cytometer (BD Biosciences, Franklin Lakes, NJ, USA). AVOs were quantified using the Cy5-Phycoerythrin emission (PE) signal detector.

GFP-microtubule-associated protein 1 light chain 3 (LC3) transfection. LC3 is involved in microtubule assembly and autophagy. Endogenous LC3 is processed into LC3-I and then lipidated to LC3-II. LC3-II is associated with the autophagosome membrane and thus regarded as a promising marker of autophagy. Ovarian cancer cells were transfected with siRNA as described previously on day 1 and then transfected with pEGFP-LC3 plasmid (kindly provided by N. Mizushima and T. Yoshimori, National Institute for Basic Biology, Okazaki, Japan) on day 2. pEGFP-LC3 was added at a final concentration of $1.6 \mu \mathrm{g} / \mathrm{ml}$ using Lipofectamine 2000 transfection reagent (Invitrogen) according to the manufacturer's instructions. Following incubation for $6 \mathrm{~h}$, cells were replenished with serum-free Opti-MEM for $12 \mathrm{~h}$. The resultant cells were fixed with $95 \%$ ethanol for microscopic analysis.

Sulphorhodamine B (SRB) assay. Cell proliferation was evaluated with SRB by measuring cellular protein content. Cells in the exponential growth phase were seeded in 96-well culture plates at a final concentration of $5 \times 10^{3}$ cells per well. After 
A

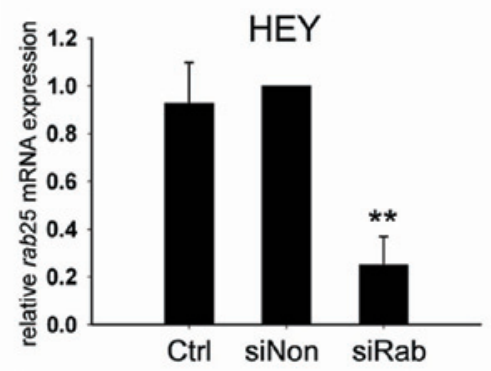

B

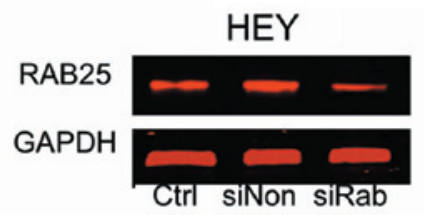

C

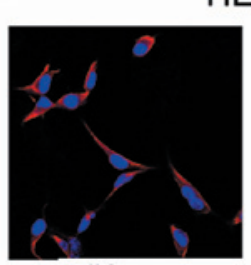

siNon
HEY

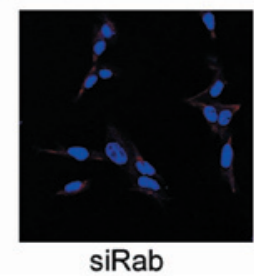

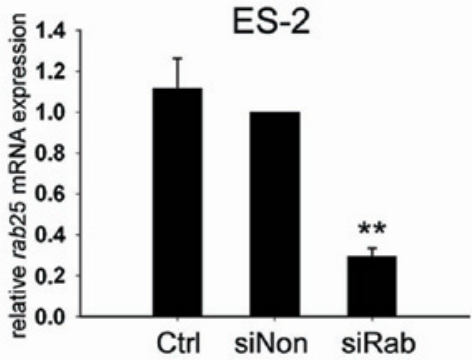

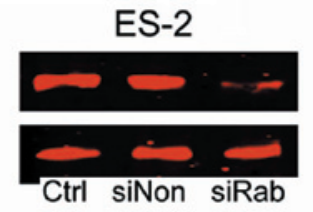

ES-2

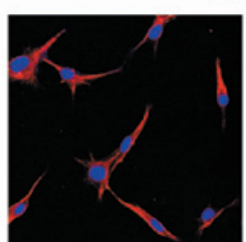

siNon

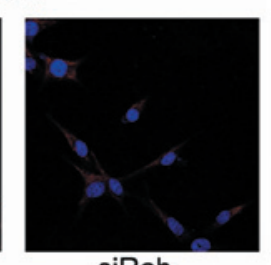

siRab

Figure 1. Effect of siRNA on RAB25 expression in ovarian cancer cells. (A) Knockdown of RAB25 inhibited the RAB25 mRNA level in HEY and ES-2 cells. Cells were transfected with non-targeting siRNA (siNon) or RAB25 siRNA (siRab). Vehicle control is also listed. The relative expression level of $R A B 25$ mRNA was analyzed using quantitative real-time PCR. GAPDH was used as an internal reference. ${ }^{* *} \mathrm{P}<0.01$ compared with siNon. (B) Western blotting verified knockdown of RAB25 in HEY and ES-2 cells $48 \mathrm{~h}$ after transfection with siRab. Equal loading was confirmed by blotting with an anti-GAPDH antibody. (C) Immunocytofluorescence showed that expression of RAB25 was decreased in the cells transfected with siRab compared with siNon. Red fluorescence represented the expression of RAB25. Blue fluorescence of DAPI nuclear staining is also shown.

$48 \mathrm{~h}$, cells were fixed with $100 \mu \mathrm{l}$ of $30 \%$ iced trichloroacetic acid at $4^{\circ} \mathrm{C}$ for $1 \mathrm{~h}$. Following washing and air-drying, $100 \mu \mathrm{l}$ of $0.4 \%(\mathrm{w} / \mathrm{v}) \mathrm{SRB}$ solution in $1 \%$ acetic acid was added into each well and incubated for $30 \mathrm{~min}$ at room temperature. Excess dye was removed by washing 5 times with $1 \%$ acetic acid and the plates were then allowed to air dry. The optical density values of resuspended SRB in $10 \mathrm{mM}$ Tris buffer were read at $570 \mathrm{~nm}$ on a microplate spectrophotometer to evaluate cell proliferation.

Cell cycle analysis. Cells were synchronized in G1-phase by serum starvation for $12 \mathrm{~h}$. The collected cells were then fixed in $70 \%$ ethanol at $4^{\circ} \mathrm{C}$ overnight. The following day, cells were washed with PBS and dyed with Propidium Iodide (PI) solution (Dingguo, Shanghai, China) for $30 \mathrm{~min}$ at room temperature in the dark. PE signals were detected by flow cytometry. Modfit 3.0 software (Verity software Inc., Topsham, ME, USA) was used for cell cycle analysis.

Apoptosis assay. Cells were harvested $48 \mathrm{~h}$ after siRNA transfection, washed twice with PBS and resuspended in $1 \mathrm{X}$ binding buffer (Invitrogen). Following incubation with Annexin V-FITC and PI staining solution (Invitrogen) at room temperature for $15 \mathrm{~min}$ in the dark, cells were analyzed immediately by flow cytometry. The signal of Annexin V-FITC was detected using the FITC-signal detector and PI with the PE signal detector. The Annexin V-FITC-positive/PI-negative population represented early apoptotic cells.
Statistical analysis. Data are expressed as the mean \pm SD obtained from three individual experiments. Statistical differences between the control and treatment groups were determined by one-way ANOVA followed by Dunnet's test. $\mathrm{P}<0.05$ was considered to indicate statistically significant differences. Representative images from western blotting and microscope analysis are shown.

\section{Results}

RAB25 siRNA downregulates the expression of RAB25 and increases the expression of pERK1/2 in ovarian cancer cells. HEY and ES-2 ovarian cancer cells were assessed for the knockdown efficiency of RAB25 siRNA $24 \mathrm{~h}$ after transfection. Transfection with siRab resulted in a $75.1 \%$ reduction of RAB25 mRNA levels in HEY cells $(\mathrm{P}<0.01)$ and a $70.7 \%$ reduction in ES-2 cells $(\mathrm{P}<0.01)$ compared with siNon-treated cells (Fig. 1A). The RAB25 protein expression in transfected cells was consistent with the transcription assay results (Fig. 1B and C). These data revealed that RAB25 was effectively downregulated by siRab in the ovarian cancer cell lines used in this study.

Knockdown of RAB25 increases AVOs through the ERK1/2 signaling pathwayinovarian cancercells. To investigate whether RAB25 is involved in autophagy, the level of AVOs was examined in transfected ovarian cancer cells. The percentages of cells with AVOs in siRab-transfected cells were significantly higher 
A

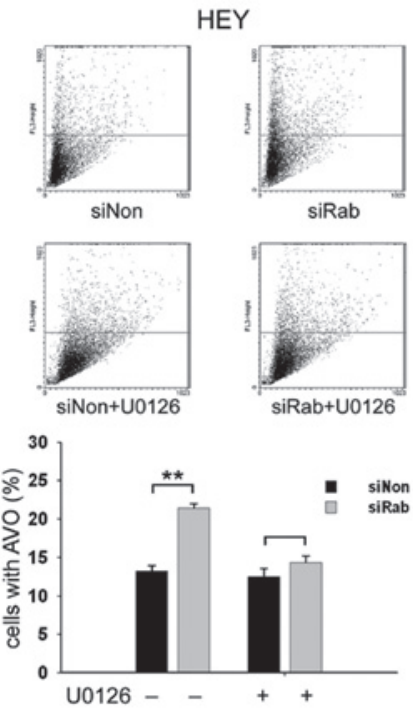

C

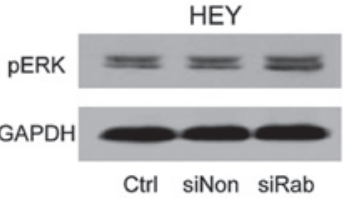

B
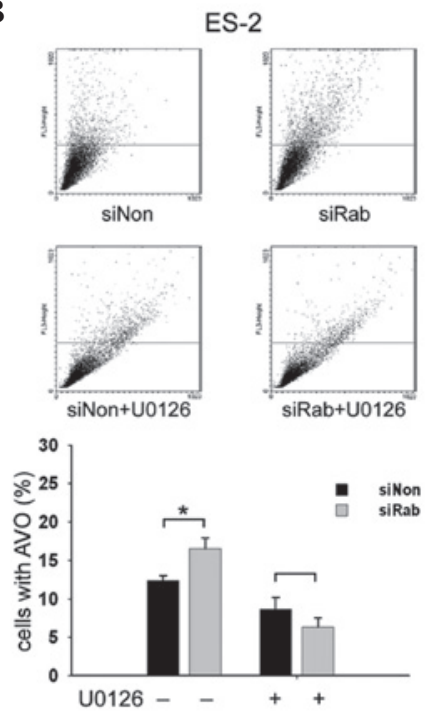

ES-2

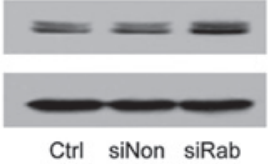

Figure 2. Effect of Rab25 siRNA on AVOs formation and related signaling pathway. (A and B) Knockdown of RAB25 increased the AVOs in ovarian cancer cells. After being transfected with siNon or siRab, (A) HEY and (B) ES-2 cells were treated with/without U0126 for $24 \mathrm{~h}$. Cells were then stained with acridine orange for flow cytometry analysis. "P $\mathrm{P}<.05$ or ${ }^{* * *} \mathrm{P}<0.01$ compared with siNon. (C) Western blotting revealed upregulation of p-ERK 1/2 in HEY and ES-2 cells $48 \mathrm{~h}$ after transfection with siRab. Equal loading was confirmed by blotting with an anti-GAPDH antibody. AVO, acidic vesicular organelle; $\mathrm{p}$-ERK, phospho-ERK, siNon, siGLO non-targeting siCONTROL siRNA; siRab, RAB25 ON-TARGET plus SMART pool siRNA.

A

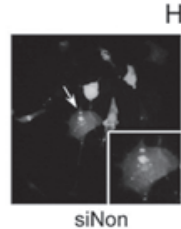

HEY
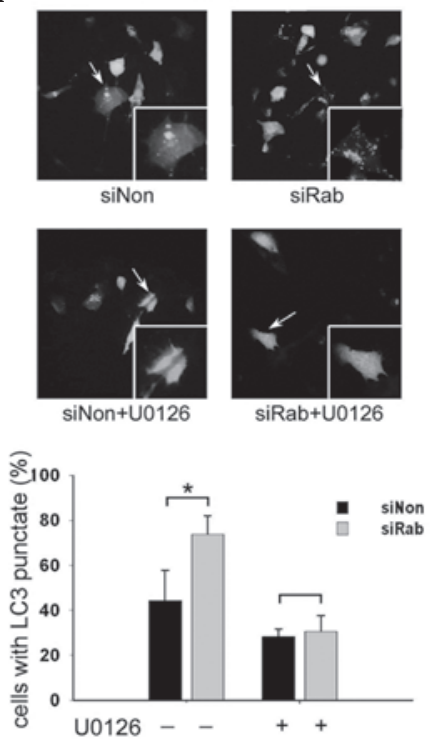

B

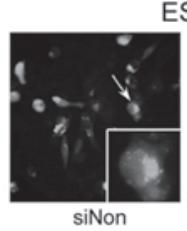

ES-2
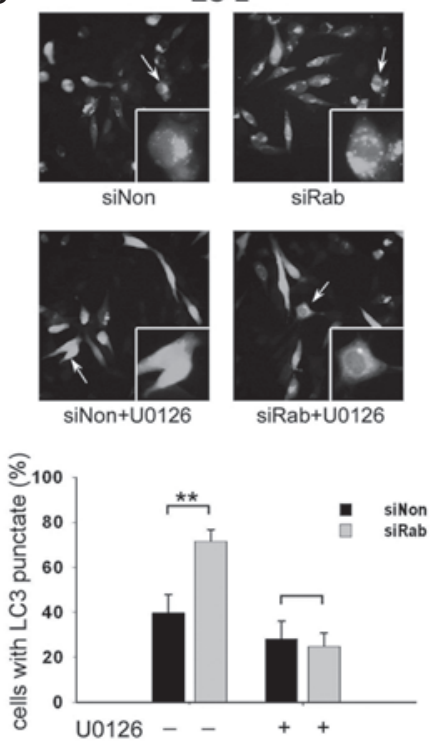

Figure 3. Effect of Rab25 siRNA on LC3 punctate fluorescence formation. Knockdown of RAB25 increased the LC3 punctuate fluorescence formation in ovarian cancer cells. (A) HEY and (B) ES-2 cells co-transfected with siRNA and pEGFP-LC3 plasmid were treated with or without U0126 for 24 h. Punctate fluorescence was observed under fluorescent microscope. ${ }^{*} \mathrm{P}<0.05$ or ${ }^{* *} \mathrm{P}<0.01$ compared with siNon. LC3, microtubule-associated protein 1 light chain 3 ; siNon, siGLO non-targeting siCONTROL siRNA; siRab, RAB25 ON-TARGET plus SMART pool siRNA.

than those in siNon-transfected cells (21.4 vs. $13.2 \%$ in $\mathrm{HEY}$ cells and 16.5 vs. $12.4 \%$ in ES-2 cells, $\mathrm{P}<0.05$; Fig. $2 \mathrm{~A})$. These data suggest that knockdown of RAB25 promotes autophagy in ovarian cancer. The ERK1/2 signaling pathway plays a role in regulating autophagy (24-27) and therefore the effect of RAB25
siRNA on the ERK signaling pathway in ovarian cancer cells was examined. Compared with siNon, transfection with siRab increased the expression of phospho-ERK1/2 (Fig. 2B). The transfected cells were then treated with U0126, a MEK 1 inhibitor, to test whether the ERK1/2 signaling pathway is involved in 
HEY

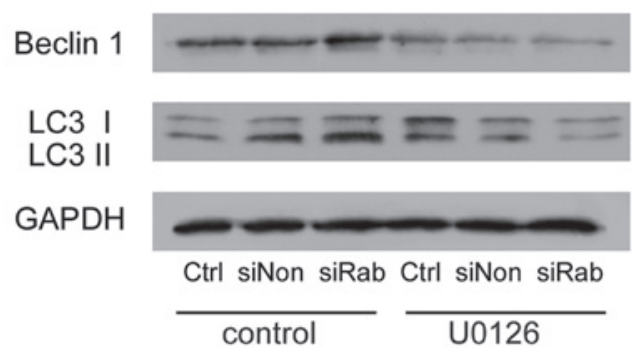

ES-2

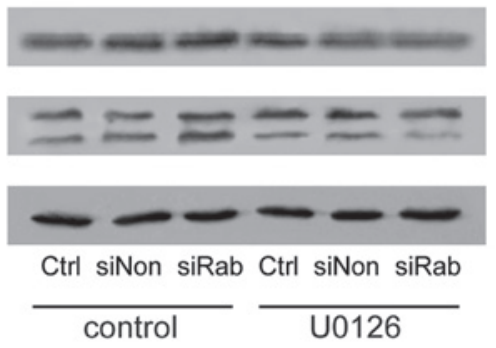

Figure 4. Knockdown of RAB25 upregulated autophagic proteins in ovarian cancer cells. Western blot assay revealed that transfection with siRab increased the expression of Beclin 1, LC3 I and LC3-II. This effect was diminished by U0126 treatment. Equal loading was confirmed by blotting with an anti-GAPDH antibody. LC3, microtubule-associated protein 1 light chain 3; siNon, siGLO non-targeting siCONTROL siRNA; siRab, RAB25 ON-TARGET plus SMART pool siRNA.

A
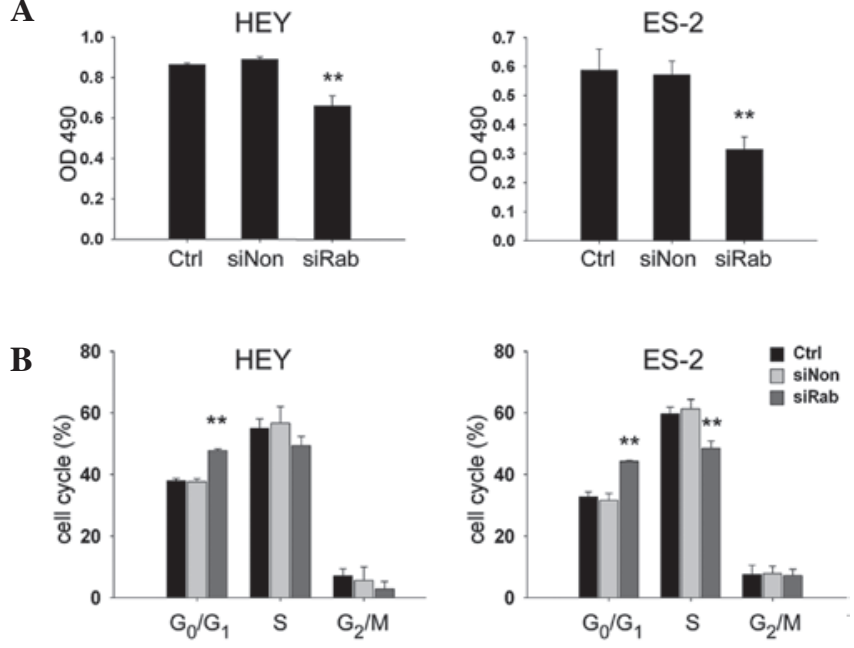

C

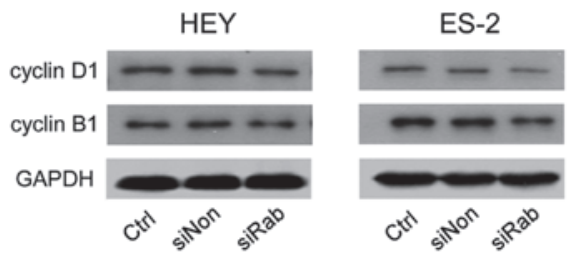

Figure 5. Knockdown of RAB25 inhibited the cell growth in ovarian cancer cells. (A) Knockdown of RAB25 blocked cell proliferation in ovarian cancer cells. After being transfected with siNon or siRab, HEY and ES-2 cells were quantified with SRB assay. ${ }^{* *} \mathrm{P}<0.01$. (B) Knockdown of RAB25 increased the proportion of cells in G0/G1 phase. HEY and ES-2 cells were transfected with siNon or siRab. Cell cycle status was detected by propidium iodide staining and flow cytometry analysis. ${ }^{* *} \mathrm{P}<0.01$. (C) Western blot assay showed that knockdown of RAB25 downregulated the expression of cyclin D1 and cyclin B1 in HEY and ES-2 cells. GAPDH was used as a loading control. siNon, siGLO non-targeting siCONTROL siRNA; siRab, RAB25 ON-TARGET plus SMART pool siRNA.

RAB25 knockdown-induced autophagy in ovarian cancer cells. Increased AVOs were noted in the untreated siRab-transfected cells but not in the U0126-treated cells (Fig. 2A), suggesting that the effect of RAB25 on autophagy is mediated through the ERK1/2 signaling pathway.

Knockdown of RAB25 increased GFP-LC3 punctate fluorescence in ovarian cancer cells. The levels of GFP-LC3 punctate fluorescence was examined in transfected ovarian cancer cells. RAB25 siRNA significantly increased the percentage of cells with GFP-LC3 punctate fluorescence in HEY (Fig. 3A) and ES-2 cells (Fig. 3B) compared with controls (44.4 vs. $73.8 \%$ in HEY and 39.8 vs. $71.7 \%$ in ES-2 cells, $\mathrm{P}<0.05$ ). It was also demonstrated that the upregulation of GFP-LC3 punctate fluorescence induced by silencing of RAB 25 was reversed by U0126 in HEY and ES-2 cells.

Knockdown of RAB25 upregulated autophagic proteins in ovarian cancer cells. Beclin 1, an autophagy-related protein, is required for the initiation of autophagy. Knockdown of RAB25 increased Beclin 1 expression and conversion of LC3-I to LC3-II. These changes were reversed by U0126 treatment (Fig. 4). These findings further confirm that RAB25 regulates autophagy through the ERK1/2 signaling pathway.

Knockdown of RAB25 inhibited cell growth in ovarian cancer cells. To determine whether knockdown of RAB25 impacted ovarian cancer cell growth, the proliferation of transfected HEY and ES-2 cells was evaluated. Transfection with siRab reduced cell growth by $25.9 \%$ in HEY cells $(\mathrm{P}<0.01)$ and $36.6 \%$ in ES-2 cells $(\mathrm{P}<0.01)$ compared with siNon (Fig. 5A). These results were further confirmed by cell cycle analysis, which revealed that siRab transfection significantly increased the proportion of cells in $\mathrm{G} 0 / \mathrm{G} 1$ phase $(\mathrm{P}<0.05)$ and decreased the proportion of cells entering $\mathrm{S}$ phase $(\mathrm{P}<0.05)$ compared with siNon (Fig. 5B). Protein expression of cyclin D1, a marker of G1/S progression and cyclin B1, a marker of G2/M progression, were analyzed by western blotting. The data indicated that siRab-transfected ovarian cancer cells exhibited lower expression of cyclin D1 and cyclin B1 compared with siNon (Fig. 5C).

Knockdown of RAB25 induced apoptosis in ovarian cancer cells. Transfected ovarian cancer cells were stained with Annexin V-FITC and PI, and the cells were then analyzed by flow cytometry to explore whether knockdown of RAB25 impacted apoptosis. Compared with siNon, siRab transfection increased the Annexin V-FITC-positive/PI-negative population in HEY (5.8 vs. $3.3 \%, \mathrm{P}<0.01$; Fig. $6 \mathrm{~A}$ ) and ES-2 cells $(8.3$ vs. $5.8 \%, \mathrm{P}<0.01$; Fig. $6 \mathrm{~B})$. These results indicated that knockdown of RAB25 increases apoptosis in ovarian cancer 
A
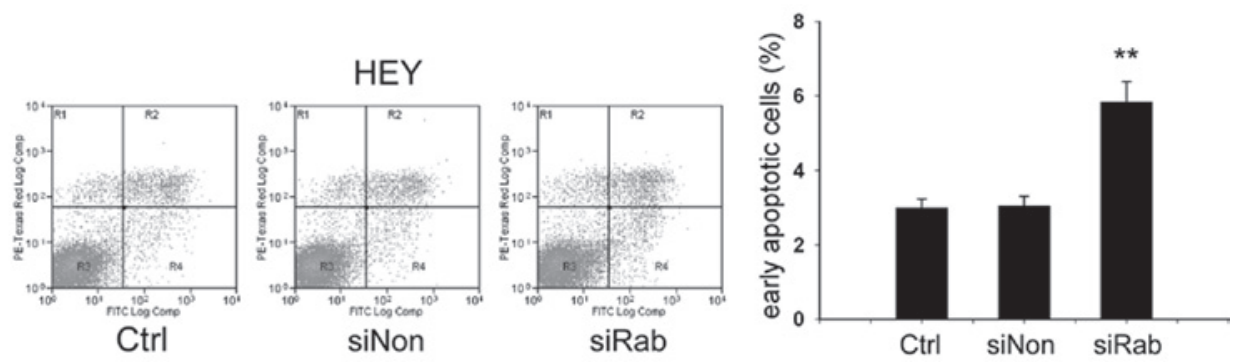

B
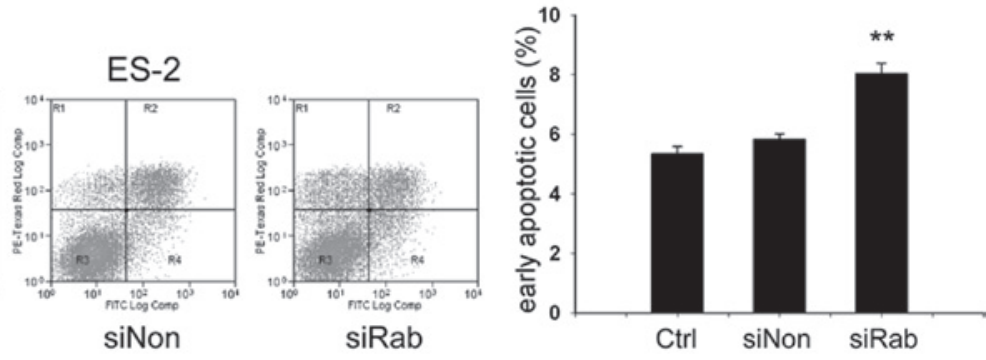

C
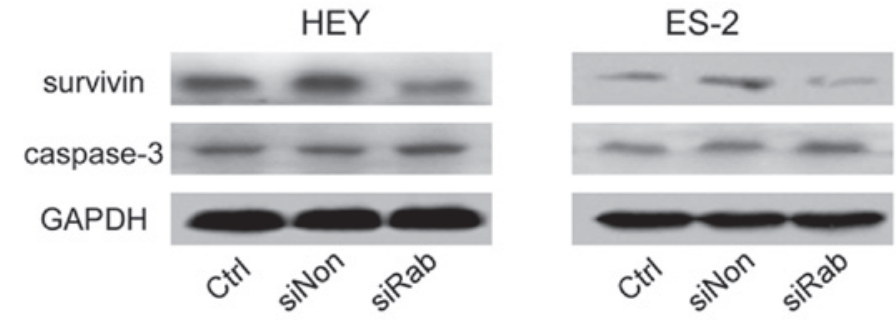

Figure 6. Knockdown of RAB25 increased apoptosis in ovarian cancer cells. (A and B) Knockdown of RAB25 increased the proportion of early apoptotic cells in ovarian cancer cells. HEY and ES-2 cells were transfected with siNon or siRab and then subjected to Annexin V-FITC/propidium iodide assay. The lower-right region represents the early apoptotic cells. ${ }^{* *} 0.01$. (C) Knockdown of RAB25 downregulated the expression of survivin and increased the level of cleaved caspase-3 in HEY and ES-2 cells. GAPDH was use as a loading control. siNon, siGLO non-targeting siCONTROL siRNA; siRab, RAB25 ON-TARGET plus SMART pool siRNA

cells. Since survivin plays a critical role in cell survival and apoptosis, the expression of survivin was analyzed, which revealed a reduction following siRab transfection. The level of cleaved caspase-3, a marker of apoptosis, was also increased by siRab transfection (Fig. 6C).

\section{Discussion}

Cancer cell transformation involves alterations in cell shape and behavior. Cancers of epithelial origin arise from a loss of polarized epithelial monolayer and adoption of migratory behaviors that lead to invasion (28). RAB25 regulates the membrane-trafficking system that changes epithelial cell polarity and aids the localization of integrin-recycling vesicles that enhance invasive ability (29), suggesting a role for RAB25 in tumorigenesis.

Overexpression of RAB25 has been reported in ovarian cancer and is associated with decreased survival rates (12). Studies on ovarian cancer have demonstrated that RAB25 facilitates cell proliferation and anti-apoptotic effects $(12,14,15)$. In agreement with previous studies, these results indicated that knockdown of RAB25 in ovarian cancer cells inhibited cell growth and induced apoptosis. Studies on RAB25 expression in other types of cancer, however, have shown mixed results. RAB25 has been reported to be overexpressed in liver and bladder cancer, but downregulated in colon cancers (28). RAB25 expression has been associated with invasiveness in breast cancer, but loss of expression has also been reported in some breast cancer tissue $(30,31)$. The role of RAB25 in tumorigenesis may be tissue-specific and requires further elucidation.

Autophagy occurs after nutrient deprivation or following chemotherapy in cancer cells $(32,33)$. The impact of autophagy on cancer progression is controversial and whether autophagic activities in cells actually cause death or are a survival mechanism remains unclear. However, excessive autophagy damages the cell. In the present study, knockdown of RAB25 promoted autophagy, suggesting that suppression of RAB25 was stressful to ovarian cancer cells and induced programmed cell death mechanisms. This result implies that RAB25 is a new candidate for targeted cancer therapy.

Previous studies have demonstrated a correlation between the MAPK/ERK pathway and autophagy (24-27). Prolonged activation and cytoplasmic sequestration of ERK1/2 induce autophagy (34-37). In this study, knockdown of RAB25 with siRNA activated the ERK1/2 pathway and promoted autophagy. Blocking ERK1/2 concurrently inhibited autophagy, suggesting that the ERK signaling pathway mediates the effects of RAB25 on autophagy in ovarian cancer cells.

In conclusion, knockdown of RAB25 promoted autophagy, inhibited cell proliferation and induced apoptosis in ovarian 
cancer cells. These results support the tumorigenic role of RAB25 in ovarian cancer cells.

\section{Acknowledgements}

This study was supported by the National Natural Science Foundation of China (grant numbers 30872755 and 81020108027 for Y. Feng) and the Shanghai Leading Academic Discipline Project (grant number B117 for Y. Feng). This research received no specific grant from any funding agency in the public, commercial, or not-for-profit sector.

\section{References}

1. Colombo N, Van Gorp T, Parma G, et al: Ovarian cancer. Crit Rev Oncol Hematol 60: 159-179, 2006.

2. Armstrong DK, Bundy B, Wenzel L, et al: Intraperitoneal cisplatin and paclitaxel in ovarian cancer. New Engl J Med 354: 34-43, 2006

3. Bristow RE, Tomacruz RS, Armstrong DK, et al: Survival effect of maximal cytoreductive surgery for advanced ovarian carcinoma during the platinum era: A meta-analysis. J Clin Oncol 20: 1248-1259, 2002.

4. Shih IeM and Davidson B: Pathogenesis of ovarian cancer: clues from selected overexpressed genes. Future Oncol 5: 1641-1657, 2009.

5. Pisano C, Bruni GS, Facchini G, et al: Treatment of recurrent epithelial ovarian cancer. Ther Clin Risk Manag 5: 421-426, 2009.

6. Nelson AE, Francis JE and Zorbas H: Population screening and early detection of ovarian cancer in asymptomatic women. Aust N Z J Obstet Gynecol 49: 448-450, 2009.

7. Marth C, Hiebl S, Oberaigner W, et al: Influence of department volume on survival for ovarian cancer: results from a prospective quality assurance program of the Austrian Association for Gynecologic Oncology. Int J Gynecol Cancer 19: 94-102, 2009.

8. Holschneider $\mathrm{CH}$ and Berek JS: Ovarian cancer: Epidemiology, biology, and prognostic factors. Semin Surg Oncol 19: 3-10, 2000.

9. Goldenring JR, Shen KR, Vaughan HD, et al: Identification of a small GTP-binding protein, RAB25, expressed in the gastrointestinal mucosa, kidney, and lung. J Biol Chem 268: 18419-18422, 1993.

10. He H, Dai F, Yu L, et al: Identification and characterization of nine novel human small GTPases showing variable expressions in liver cancer tissues. Gene Expr 10: 231-242, 2002.

11. Mor O, Nativ O, Stein A, et al: Molecular analysis of transitional cell carcinoma using cDNA microarray. Oncogene 22: 7702-7710, 2003.

12. Cheng KW, Lahad JP, Kuo WL, et al: The RAB25 small GTPase determines aggressiveness of ovarian and breast cancers. Nat Med 10: 1251-1256, 2004.

13. Caswell PT, Spence HJ, Parsons M, et al: Rab25 associates with alpha 5 beta 1 integrin to promote invasive migration in $3 \mathrm{D}$ microenvironments. Dev Cell 13: 496-510, 2007.

14. Cheng KW, Lu YL and Mills GB: Assay of Rab25 function in ovarian and breast cancers. Method Enzymol 403: 202-215, 2005.

15. Fan Y, Xin XY, Chen BL, et al: Knockdown of Rab25 expression by RNAi inhibits growth of human epithelial ovarian cancer cells in vitro and in vivo. Pathology 38: 561-567, 2006.

16. Tsujimoto Y and Shimizu S: Another way to die: autophagic programmed cell death. Cell Death Differ 12: 1528-1534, 2005.

17. Lum JJ, Bauer DE, Kong M, et al: Growth factor regulation of autophagy and cell survival in the absence of apoptosis. Cell 120: 237-248, 2005
18. Ogier-Denis E and Codogno P: Autophagy: a barrier or an adaptive response to cancer. Biochim Biophys Acta 1603: 113-128, 2003.

19. Qu XP, Yu J, Bhagat G, et al: Promotion of tumorigenesis by heterozygous disruption of the beclin 1 autophagy gene. J Clin Invest 112: 1809-1820, 2003.

20. Mathew R, Karp CM, Beaudoin B, et al: Autophagy suppresses tumorigenesis through elimination of p62. Cell 137: 1062-1075, 2009.

21. Groth-Pedersen L, Ostenfeld MS, Hoyer-Hansen M, et al: Vincristine induces dramatic lysosomal changes and sensitizes cancer cells to lysosome-destabilizing siramesine. Cancer Res 67: 2217-2225, 2007.

22. Ulasov IV, Sonabend AM, Nandi S, et al: Combination of adenoviral virotherapy and temozolomide chemotherapy eradicates malignant glioma through autophagic and apoptotic cell death in vivo. Br J Cancer 100: 1154-1164, 2009.

23. Paglin S, Hollister T, Delohery T, et al: A novel response of cancer cells to radiation involves autophagy and formation of acidic vesicles. Cancer Res 61: 439-444, 2001.

24. Wu WK, Cho CH, Lee CW, et al: Macroautophagy and ERK phosphorylation counteract the antiproliferative effect of proteasome inhibitor in gastric cancer cells. Autophagy 6: 228-238, 2010.

25. Mujumdar N, Mackenzie TN, Dudeja V, et al: Triptolide induces cell death in pancreatic cancer cells by apoptotic and autophagic pathways. Gastroenterology 139: 598-608, 2010.

26. Wang J, Whiteman MW, Lian H, et al: A non-canonical MEK/ ERK signaling pathway regulates autophagy via regulating Beclin 1. J Biol Chem 284: 21412-21424, 2009.

27. Subramaniam S and Unsicker K: Extracellular signal-regulated kinase as an inducer of non-apoptotic neuronal death. Neuroscience 138: 1055-1065, 2006.

28. Goldenring JR and Nam KT: Rab25 as a tumour suppressor in colon carcinogenesis. Br J Cancer 104: 33-36, 2011.

29. Caswell PT, Spence HJ, Parsons M, et al: Rab25 associates with alpha5beta1 integrin to promote invasive migration in 3D microenvironments. Dev Cell 13: 496-510, 2007.

30. Cheng JM, Volk L, Janaki DK, et al: Tumor suppressor function of Rab25 in triple-negative breast cancer. Int J Cancer 126: 2799-2812, 2009

31. Cheng JM, Ding M, Aribi A, et al: Loss of RAB25 expression in breast cancer. Int J Cancer 118: 2957-2964, 2006.

32. Chatterjee SJ and Pandey S: Chemo-resistant melanoma sensitized by tamoxifen to low dose curcumin treatment through induction of apoptosis and autophagy. Cancer Biol Ther 11: 216-228, 2011.

33. Schnekenburger M, Grandjenette C, Ghelfi J, et al: Sustained exposure to the DNA demethylating agent, 2'-deoxy-5-azacytidine, leads to apoptotic cell death in chronic myeloid leukemia by promoting differentiation, senescence, and autophagy. Biochem Pharmacol 81: 364-378, 2011.

34. Cagnol S and Chambard JC: ERK and cell death: mechanisms of ERK-induced cell death-apoptosis, autophagy and senescence. FEBS J 277: 2-21, 2010.

35. Mebratu Y and Tesfaigzi Y: How ERK1/2 activation controls cell proliferation and cell death: Is subcellular localization the answer? Cell Cycle 8: 1168-1175, 2009.

36. Glading A, Koziol JA, Krueger J, et al: PEA-15 inhibits tumor cell invasion by binding to extracellular signal-regulated kinase 1/2. Cancer Res 67: 1536-1544, 2007.

37. Mebratu YA, Dickey BF, Evans C, et al: The BH3-only protein $\mathrm{Bik} / \mathrm{Blk} / \mathrm{Nbk}$ inhibits nuclear translocation of activated ERK1/2 to mediate IFNgamma-induced cell death. J Cell Biol 183: 429-439, 2008 . 